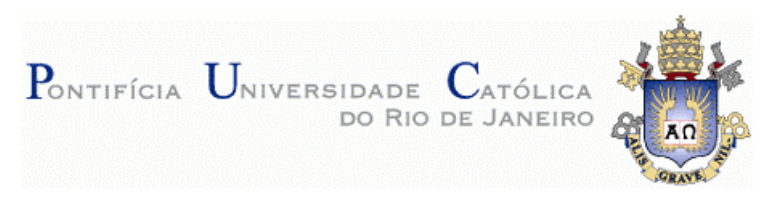

Guilherme Pinto Guimarães

\title{
Uma Formulação de Elementos Finitos Axissimétricos para Análise de Tubos Laminados em Materiais Compósitos
}

\section{Dissertação de Mestrado}

Dissertação apresentada como requisito parcial para obtenção do título de Mestre pelo Programa de PósGraduação em Engenharia Mecânica da PUC-Rio.

Orientador: Prof. Carlos Alberto de Almeida 


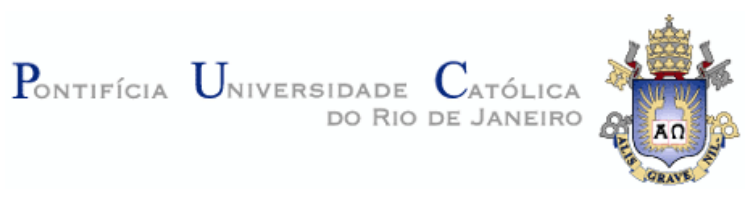

Guilherme Pinto Guimarães

\section{Uma Formulação de Elementos Finitos Axissimétricos para Análise de Tubos Laminados em Materiais Compósitos}

Dissertação apresentada como requisito parcial para obtenção do título de Mestre pelo Programa de Pós-Graduação em Engenharia Mecânica da PUC-Rio. Aprovada pela Comissão Examinadora abaixo assinada.

\section{Carlos Alberto de Almeida}

Orientador

Pontifícia Universidade Católica do Rio de Janeiro

Arthur Martins Barbosa Braga Pontifícia Universidade Católica do Rio de Janeiro

Paulo Roberto Rocha Aguiar

Centro Tecnológico do Exército

José Eugenio Leal

Coordenador Setorial do Centro Técnico Científico - PUC-Rio 
Todos os direitos reservados. É proibida a reprodução total ou parcial do trabalho sem autorização da Universidade, do Autor e do Orientador.

\section{Guilherme Pinto Guimarães}

Graduou-se em Engenharia Mecânica e de Armamento no IME - Instituto Militar de Engenharia, em 1997. Trabalha desde 1998 no CTEx - Centro Tecnológico do Exército, como pesquisador em projetos de materiais de emprego militar e como analista estrutural em modelagens e simulações por elementos finitos.

Ficha Catalográfica

Guimarães, Guilherme Pinto

Uma Formulação de Elementos Finitos Axissimétricos para Análise de Tubos Laminados em Materiais Compósitos / Guilherme Pinto Guimarães; Orientador: Carlos Alberto de Almeida - Rio de Janeiro : PUC-Rio, Departamento de Engenharia Mecânica, 2006.

120 f. : il. ; $30 \mathrm{~cm}$

1. Dissertação (mestrado) - Pontifícia Universidade Católica do Rio de Janeiro, Departamento de Engenharia Mecânica.

Inclui referências bibliográficas.

1. Engenharia Mecânica - Teses. 2. Elementos Finitos. 3. Materiais Compósitos. 4. Estrutura Laminada. 5. Modelo Axissimétrico. I. de Almeida, Carlos Alberto. II. Pontifícia Universidade Católica do Rio de Janeiro. Departamento de Engenharia Mecânica. III. Título. 


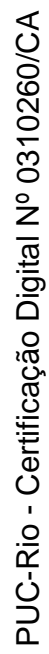

A Deus,

a Roberta,

e a meus pais, Roney e José Custódio. 


\section{Agradecimentos}

À minha mulher, Roberta, por tudo.

Aos meus pais, Roney e José Custódio, pelo incentivo e pelo apoio.

Ao professor, orientador e amigo Carlos Alberto de Almeida, pela confiança em mim depositada, pela atenção, pelo trabalho ao longo destes anos, e, sobretudo, por ser um exemplo de profissional dedicado.

Ao colega de trabalho e amigo, Paulo Roberto, pelo incentivo, pelo apoio e por discussões técnicas de valor.

Ao colega de trabalho e amigo, Marcelo Brisola, pelo incentivo, pelo apoio e pela realização dos desenhos técnicos em CAD, neste trabalho.

Aos colegas do programa de pós-graduação em engenharia mecânica da PUCRio e amigos, Abel Andrews e Anderson Pereira, pelo apoio, incentivo e colaborações em discussões técnicas.

Aos demais professores e funcionários do Departamento de Engenharia Mecânica da PUC-Rio, pelos ensinamentos e apoio. 


\section{Resumo}

Guimarães, Guilherme Pinto. Uma Formulação de Elementos Finitos Axissimétricos para Análise de Tubos Laminados em Materiais Compósitos. Rio de Janeiro, 2006. 120p. Dissertação de Mestrado Departamento de Engenharia Mecânica, Pontifícia Universidade Católica do Rio de Janeiro.

O emprego de materiais compósitos em estruturas tem ganhado importância na prática da engenharia devido às suas características de alta resistência mecânica, baixa densidade e boa estabilidade a efeitos térmicos. Uma das classes de compósitos, a de laminados fibrosos, pode ser utilizada em tubulações sujeitas às diversas formas de carregamentos, como pressão interna e/ou externa, tração longitudinal, torção, temperatura, etc. O presente Trabalho tem por objetivo propor, implementar e testar a formulação de um modelo de elemento finito axissimétrico, para a representação do comportamento de um tubo laminado por camadas de materiais compósitos fibrosos. A modelagem consiste em representar a seção geratriz de um tubo cilíndrico por um elemento quadrilateral de quatro nós, com três graus de liberdade por nó, com os deslocamentos nodais tomados em relação aos eixos de um sistema cilíndrico de coordenadas. Considera-se a perfeita adesão das camadas, garantindo a continuidade do campo de deslocamentos. Modelos constitutivos de materiais com o comportamento ortotrópico e/ou o transversalmente isotrópico foram implementados, obtendo-se respostas para os campos de deslocamentos, de deformações e de tensões atuantes. $\mathrm{Na}$ validação do modelo numérico, considerou-se a comparação de seus resultados com os de soluções analíticas, disponíveis na literatura, e aqueles fornecidos por um programa comercial de elementos finitos, empregando o modelo com elementos sólidos. Foram propostos, para os testes em ambos os casos, exemplos de laminados com uma a quatro camadas, com fibras orientadas em diferentes ângulos. Destas comparações, verifica-se uma boa convergência das soluções numéricas obtidas com o presente modelo, representativo das principais características cinemáticas da classe de problemas representada.

\section{Palavras-chave}

Elementos finitos; Materiais compósitos; Estrutura laminada; Modelo Axissimétrico. 


\section{Abstract}

Guimarães, Guilherme Pinto. Uma Formulação de Elementos Finitos Axissimétricos para Análise de Tubos Laminados em Materiais Compósitos. Rio de Janeiro, 2006. 120p. Dissertação de Mestrado Departamento de Engenharia Mecânica, Pontifícia Universidade Católica do Rio de Janeiro.

The use of composite materials in structures has grown in the engineering practice due to its characteristics, of high strength, low density and a good stability to thermal effects. A class of composites, the fibrous laminates, is generally used in tubes subjected to many types of loadings as internal and/or external pressure, traction, torsion, temperatures, etc. This work has the objectives to propose, implement and test an axisymmetric finite element model formulation that represents the mechanical behavior of a fibrous laminated composite tube. Modeling consists in representing the cylindrical tube generating section by a quadrilateral element with four nodes and three degrees-of-freedom per node, with three nodal displacements defined in a cylindrical coordinate system. Layers are considered perfectly bonded together, assuring continuity between elements on the displacement fields. Orthotropic and/or transverse isotropic constitutive material models were implemented, allowing solutions for displacement, strain and stress fields. In the element numerical model validation, result comparisons with those from analytical solutions available on literature and those from the use of layered solid elements in a commercial finite element program were considered. Some examples, considering one to four layers, with different fiber angles, were proposed for model testing. It is noted a good numerical convergence for the presenting model solutions which represent the main kinematic characteristics for this class of problems.

\section{Keywords}

Finite Elements; Composite Materials; Laminated Structure; Axisymmetric Model. 


\section{Sumário}

1. Introdução

2. Materiais Compósitos $\quad 21$

2.1. Classificação de Materiais Compósitos 22

2.2. Compósitos Fibrosos $\quad 24$

2.3. Comportamento Mecânico de Materiais Compósitos Laminados - 25 Análise Macromecânica

2.3.1. Modelos de Material 25

2.3.2. Constantes de Engenharia 28

2.3.3. Relações Constitutivas em Coordenadas Cilíndricas 30

2.3.4. Propriedades de Compósitos Fibrosos Laminados Comerciais 33

3. Implementação do Método de Elementos Finitos 35

3.1. Formulação Variacional e Princípio dos Trabalhos Virtuais 35

3.2. Definição do Problema de Tubos Cilíndricos 42

3.3. Proposta de Elemento Finito 42

3.4. Malha de Elementos Finitos $\quad 48$

3.5. Definição de Condições de Contorno - Carregamentos e Restrições 50 de Graus de Liberdade

3.6. Implementação em Ferramenta Computacional 51

4. Soluções Analíticas

4.1. Desenvolvimento 52

4.2. Implementação para Cálculo das Soluções Analíticas 62

5. Testes Numéricos 64

5.1. Considerações Iniciais 64

5.2. Definições Preliminares para os Testes 66

5.3. Propostas de Exemplos para Testes Numéricos - Condições de 68 Contorno e Empilhamentos de Laminados

5.3.1. Condições de Contorno 68 
5.3.2. Empilhamentos dos Laminados $\quad 72$

5.4. Testes de Comparação com a Solução Analítica 72

5.4.1. Laminado $\left[0^{\circ}\right] \quad 73$

5.4.2. Laminado $\left[+90^{\circ}\right] \quad 80$

5.4.3. Laminado $\left[+45^{\circ}\right] \quad 84$

5.4.4. Laminado $\left[+45^{\circ} /-45^{\circ}\right] \quad 89$

5.4.4. Laminado $\left[0 \%+90^{\circ} /+90^{\circ} / 0^{\circ}\right]$

5.4.6. Laminado [ $\left.+45^{\circ} /-45^{\circ} /-45^{\circ} / 45^{\circ}\right]$

5.5. Testes de Comparação com Modelagens Sólidas no Programa 101 ANSYS $^{\circledR}$

5.5.1. Laminado $\left[+45^{\circ}\right] \quad 102$

5.5.2. Laminado $\left[+45^{\circ} /-45^{\circ}\right] \quad 109$

$\begin{array}{ll}\text { 6. Conclusões } & 117\end{array}$

7. Referências Bibliográficas 120 


\section{Lista de Tabelas}

Tabela 2.1: Algumas Propriedades Mecânicas de Materiais Compósitos 34 Fibrosos

Tabela 5.1: Dados Numéricos de Entrada - Compósito T300/5208 67

Tabela 5.2: Empilhamentos Propostos e Condições de Contorno Impostas 72 


\section{Lista de Figuras}

Figura 2.1: Exemplo de Arranjo de Fibras em Matriz para Compósito

Fibroso

Figura 2.2: Definição de Planos de Simetria para o Comportamento

Mecânico de um Corpo no Espaço

Figura 2.3: Definição da Estrutura e Sistemas de Coordenadas

Figura 3.1: Corpo Volumétrico Sujeito a Carregamentos e a Restrições de 38 Movimento

Figura 3.2: Proposta de Elemento Finito Axissimétrico de 04 Nós 43

Figura 3.3: Exemplo de Malha de Elementos Finitos Representando a 49 Seção do Tubo Cilíndrico - Discretização Radial

Figura 5.1: Esquemas de Algumas Orientações de Fibras 67

Figura 5.2: Condições de Contorno Adotadas - Tipos (1) e (2) 70

Figura 5.3: Exemplo de Vinculação de Graus de Liberdade 71

Circunferenciais

Figura 5.4: Convergência do Deslocamento Radial / Raio Interno - [0 $] 74$

Figura 5.5: Comparação de Deslocamentos Radiais - $\left[0^{\circ}\right]$

Figura 5.6: Comparação de Deformações Circunferenciais $\left(\varepsilon_{\theta \theta}\right)-\left[0^{\circ}\right] \quad 75$

Figura 5.7: Convergência de Deformações Radiais $\left(\varepsilon_{\mathrm{RR}}\right)-\left[0^{\circ}\right] \quad 76$

Figura 5.8: Comparação de Deformações Radiais $\left(\varepsilon_{R R}\right)-\left[0^{\circ}\right] \quad 76$

Figura 5.9: Tensões Axiais ao Longo da Espessura - [ $\left.0^{\circ}\right]$

Figura 5.10: Tensões Circunferenciais ao Longo da Espessura - [ $\left.0^{\circ}\right] \quad 77$

Figura 5.11: Tensões Radiais ao Longo da Espessura - [ $\left.0^{\circ}\right]$

Figura 5.12: Comparação de Deformações Radiais (16 Elementos) - [ $0^{\circ}$ ] 79

Figura 5.13: Comparação de Tensões Radiais (16 Elementos) - [ $0^{\circ}$ ] 79

Figura 5.14: Convergência para Deslocamentos Axiais $(u)-\left[+90^{\circ}\right] \quad 80$

Figura 5.15: Comparação de Deslocamentos Radiais $(w)-\left[+90^{\circ}\right] \quad 80$

Figura 5.16: Deformações Circunferenciais $-\left[+90^{\circ}\right]$

Figura 5.17: Deformações Radiais $-\left[+90^{\circ}\right] \quad 82$

Figura 5.18: Tensões Axiais - $\left[+90^{\circ}\right]$ 
Figura 5.19: Tensões Circunferenciais $-\left[+90^{\circ}\right]$

Figura 5.20: Tensões Radiais $-\left[+90^{\circ}\right]$

Figura 5.21: Convergência de Deslocamentos Axiais - $\left[+45^{\circ}\right]$

Figura 5.22: Deslocamentos Circunferenciais $-\left[+45^{\circ}\right]$

Figura 5.23: Deslocamentos Radiais $-\left[+45^{\circ}\right]$

Figura 5.24: Deformações Circunferenciais $-\left[+45^{\circ}\right]$

Figura 5.25: Deformações Radiais $-\left[+45^{\circ}\right]$

Figura 5.26: Deformações Angulares $\left(\gamma_{x_{\theta}}\right)-\left[+45^{\circ}\right]$

Figura 5.27: Tensões Axiais - [+45 $]$

Figura 5.28: Tensões Circunferenciais $-\left[+45^{\circ}\right]$

Figura 5.29: Tensões Radiais $-\left[+45^{\circ}\right] \quad 88$

Figura 5.30: Tensões Cisalhantes $\left(\tau_{X \theta}\right)-\left[+45^{\circ}\right] \quad 88$

Figura 5.31: Deformações Radiais $-\left[+45^{\circ}\right]-$ Modelo com 08 Elementos 89

Figura 5.32: Tensões Radiais $-\left[+45^{\circ}\right]-$ Modelo com 08 Elementos 89

Figura 5.33: Convergência de Deslocamentos Axiais - $\left[+45^{\circ} /-45^{\circ}\right] \quad 90$

Figura 5.34: Deslocamentos Circunferenciais $-\left[+45^{\circ} /-45^{\circ}\right] \quad 90$

Figura 5.35: Deslocamentos Radiais $-\left[+45^{\circ} /-45^{\circ}\right] \quad 91$

Figura 5.36: Deformações Circunferenciais $-\left[+45^{\circ} /-45^{\circ}\right] \quad 91$

Figura 5.37: Deformações Radiais - [+45\% $\left./ 45^{\circ}\right] \quad 92$

Figura 5.38: Deformações Angulares $\left(\gamma_{x \theta}\right)-\left[+45^{\circ} /-45^{\circ}\right] \quad 92$

Figura 5.39: Tensões Axiais - $\left[+45^{\circ} /-45^{\circ}\right] \quad 93$

Figura 5.40: Tensões Circunferenciais $-\left[+45^{\circ} /-45^{\circ}\right] \quad 93$

Figura 5.41: Tensões Radiais - [+45\% $\left./-45^{\circ}\right] \quad 94$

Figura 5.42: Tensões Cisalhantes $\left(\tau_{\theta R}\right)-\left[+45^{\circ} /-45^{\circ}\right] \quad 94$

Figura 5.43: Deslocamentos Radiais $-\left[0^{\circ} \%+90^{\circ} /+90^{\circ} / 0^{\circ}\right] \quad 95$

Figura 5.44: Deformações Circunferenciais $-\left[0^{\circ} \%+90 \%+90^{\circ} / 0^{\circ}\right] \quad 96$

Figura 5.45: Deformações Radiais - $\left[0^{\circ} /+90^{\circ} /+90^{\circ} / 0^{\circ}\right]$

Figura 5.46: Tensões Axiais - $\left[0 \%+90^{\circ} \%+90^{\circ} / 0^{\circ}\right] \quad 97$

Figura 5.47: Tensões Circunferenciais $-\left[0 \%+90^{\circ} \%+90^{\circ} / 0^{\circ}\right] \quad 97$

Figura 5.48: Tensões Radiais - $\left[0 \%+90^{\circ} \%+90^{\circ} 0^{\circ}\right] \quad 97$

Figura 5.49: Deslocamentos Radiais $-\left[+45^{\circ} /-45^{\circ} /-45^{\circ} /+45^{\circ}\right]$

Figura 5.50: Deformações Circunferenciais - $\left[+45^{\circ} /-45^{\circ} /-45 \%+45^{\circ}\right] \quad 99$

Figura 5.51: Deformações Radiais - $\left[+45^{\%} /-45^{\circ} /-45 \%+45^{\circ}\right]$

Figura 5.52: Tensões Axiais - $\left[+45^{\circ} /-45^{\circ} /-45^{\circ} /+45^{\circ}\right]$

Figura 5.53: Tensões Circunferenciais $-\left[+45^{\circ} /-45^{\circ} /-45^{\circ} /+45^{\circ}\right] \quad 100$

Figura 5.54: Tensões Radiais - $\left[+45 \%-45^{\circ} /-45^{\%} / 45^{\circ}\right]$ 
Figura 5.55: Tensões Cisalhantes $\left(\tau_{x \theta}\right)-\left[+45^{\circ} /-45^{\circ} /-45^{\circ} /+45^{\circ}\right] \quad 101$

Figura 5.56: Malha Sólida para Modelagem do Laminado [+45\% $\left./ 45^{\circ}\right] \quad 102$

Figura 5.57: Deslocamentos Axiais -[+45º]- Cond. de Contorno (1) e (2) 103

Figura 5.58: Deslocamentos Circunferenciais - $\left[+45^{\circ}\right]$ - Cond. de Cont. (1) 103 e (2)

Figura 5.59: Deslocamentos Radiais - [+45 ] - Cond. de Contorno (1) e (2) 104

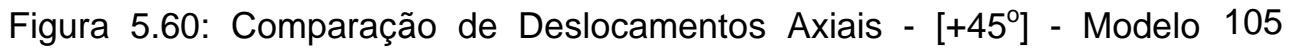
Axissimétrico com 08 Elementos Radiais - Cond. de Contorno (1) e (2)

Figura 5.61: Deformações Axiais -[+45º]- Condições de Contorno (1) e (2) 105 Figura 5.62: Deformações Circunferenciais -[+45º]- Cond. de Cont. (1) e (2) 105 Figura 5.63: Deformações Radiais -[+45º]- Condições de Contorno (1) e (2) 106 Figura 5.64: Deformações Angulares $\left(\gamma_{x_{\theta}}\right)-\left[+45^{\circ}\right]$ - Cond. de Cont. (1) e (2) 106 Figura 5.65: Tensões Axiais - $\left[+45^{\circ}\right]$ - Condições de Contorno (1) e (2) 107

Figura 5.66: Tensões Circunferenciais $-\left[+45^{\circ}\right]$ - Cond. de Contorno (1) e (2) 107

Figura 5.67: Tensões Radiais - $\left[+45^{\circ}\right]$ - Condições de Contorno (1) e (2) 108

Figura 5.68: Tensões Cisalhantes $\left(\tau_{\theta R}\right)-\left[+45^{\circ}\right]-$ Cond. de Contorno (1) e (2) 108

Figura 5.69: Deslocamentos Axiais -[+45\%-45]- Cond de Contorno (1) e (2) 109

Figura 5.70: Desl. Circunferenciais $-\left[+45^{\circ} /-45^{\circ}\right]-$ Cond. de Cont. (1) e (2) 110

Figura 5.71: Deslocamentos Radiais - [+45\% $\left./ 45^{\circ}\right]$ - Cond. de Cont. (1) e (2) 110

Figura 5.72: Deformações Axiais - $\left[+45^{\circ} /-45^{\circ}\right]$ - Cond. de Contorno (1) e (2) 111

Figura 5.73: Def. Circunferenciais - [+45\% $\left.-45^{\circ}\right]-$ Cond. de Cont. (1) e (2) 112

Figura 5.74: Deformações Radiais - [+45\%-45 $]$ - Cond. de Cont. (1) e (2) 112

Figura 5.75: Deformações Angulares $\left(\gamma_{\times \theta}\right)-\left[+45^{\circ} /-45^{\circ}\right]-$ Cond. de Cont. 112 (1) e (2)

Figura 5.76: Def. Angulares $\left(\gamma_{\times R}\right)-\left[+45^{\circ} /-45^{\circ}\right]-$ Cond. de Cont. (1) e (2) 113

Figura 5.77: Deformações Angulares $\left(\gamma_{\theta R}\right)-\left[+45^{\circ} /-45^{\circ}\right]-$ Cond. de Cont. 113 (1) e (2)

Figura 5.78: Tensão Axial - [+45\%-45 $]$ - Condições de Contorno (1) e (2) 114 Figura 5.79: Tensão Circunferencial - [+45\%-45 ] - Cond. de Cont. (1) e (2) 114 Figura 5.80: Tensão Radial - [+45\%-45 ] - Condições de Contorno (1) e (2) 115 Figura 5.81: Tensão Cisalhante $\left(\tau_{x \theta}\right)$ - [+45\% $\left./ 45^{\circ}\right]$ - Cond. de Cont. (1) e (2) 115 Figura 5.82: Tensão Cisalhante $\left(\tau_{X R}\right)$ - [+45\%-45 $]$ - Cond. de Cont. (1) e (2) 116 Figura 5.83: Tensão Cisalhante $\left(\tau_{\theta R}\right)-\left[+45^{\circ} /-45^{\circ}\right]$ - Cond. de Cont. (1) e (2) 116 


\section{Lista de Símbolos}

$A_{1}, A_{2}, E, F, F_{1}, G_{1}, K_{1}, K_{2} \quad$ Constantes de integração

$B \quad$ Matriz de deformação-deslocamento

C Matriz constitutiva do material

$C_{A m} \quad$ Matriz de amortecimento

$C_{i j} \quad$ Componente da matriz constitutiva

esp Espessura do cilindro

$E_{i i} \quad$ Módulo de elasticidade segundo a direção i

$f^{c p} \quad$ Forças de corpo

$f^{S} \quad$ Forças de superfície

$F_{c}^{i} \quad$ Forças concentradas

$G_{i j} \quad$ Módulos de cisalhamento

$h_{i} \quad$ Funções de forma

$H^{(i)} \quad$ Matriz de interpolação para o elemento (i)

J Matriz jacobiana de transformação

$K \quad$ Matriz de rigidez

M Matriz de massa

$M_{i} \quad$ Matrizes de transformação

$m \quad \cos (\phi)$

$n \quad \operatorname{sen}(\phi)$

$P_{i} \quad$ Pressão Interna

$r$, $s \quad$ Coordenadas cartesianas locais elementares

$R_{i} \quad$ Raio interno do cilindro

$R_{e} \quad$ Raio externo do cilindro

$R \quad$ Vetor de carregamentos globais

dS Elemento diferencial de área

$S \quad$ Matriz de complacência

$S_{A p} \quad$ Superfície de apoio com restrição de graus de liberdade

$S_{i j} \quad$ Coeficientes da matriz de complacência

$T \quad$ Matriz de transformação de rotação

$u^{(i)} \quad$ Vetor de deslocamentos para o elemento (i)

$U \quad$ Vetor de deslocamentos 
$\hat{U} \quad$ Vetor de deslocamentos nodais

$u \quad$ Deslocamento na direção axial $X$

$v \quad$ Deslocamento na direção circunferencial $\theta$

w Deslocamento na direção radial $\mathrm{R}$

$d V \quad$ Elemento diferencial de volume

$X-Y-Z$ Coordenadas cartesianas locais

$X-\theta-R$ Coordenadas cilíndricas

\section{Símbolos gregos}

$\varepsilon \quad$ Matriz de deformações infinitesimais

$\varepsilon_{i j} \quad$ Componente de deformação infinitesimal

$\varepsilon_{i} \quad$ Componente de tensão em notação reduzida

$\varepsilon_{x x}^{0} \quad$ Deformação uniforme

$\varepsilon_{X X} \quad$ Componente de tensão na direção $X$

$\varepsilon_{\theta \theta} \quad$ Componente de tensão na direção $\theta$

$\varepsilon_{R R} \quad$ Componente de tensão na direção R

$\phi \quad$ Ângulo de bobinamento da fibra em relação ao eixo axial

$\gamma^{0}$ Ângulo de torção do tubo

$\gamma_{i j} \quad$ Componente de deformação angular

$\gamma \times \theta \quad$ Componente de deformação angular segundo as direções $\mathrm{X}$ e $\theta$

$\gamma_{X R} \quad$ Componente de deformação angular segundo as direções $X$ e $R$

$\gamma_{\theta R} \quad$ Componente de deformação angular segundo as direções $\theta$ e $R$

$\kappa \quad$ Parâmetro de amortecimento

$\lambda, \Gamma, \Omega \quad$ Parâmetros definidos a partir de coeficientes da matriz constitutiva

$v_{i j} \quad$ Coeficientes de poisson

$\Pi \quad$ Funcional da energia potencial total

$\rho \quad$ Densidade

$\sigma \quad$ Matriz tensorial

$\sigma_{i} \quad$ Componente de tensão em notação reduzida

$\sigma_{i j} \quad$ Componente de tensão 

$\sigma_{X X} \quad$ Componente de tensão na direção $X$
$\sigma_{\theta \theta} \quad$ Componente de tensão na direção $\theta$
$\sigma_{R R} \quad$ Componente de tensão na direção $\mathrm{R}$
$\sigma_{i j} \quad$ Componente de tensão normal na direção ij
$\tau_{i j} \quad$ Componente de tensão de cisalhamento
$\tau_{X \theta} \quad$ Componente de tensão cisalhante segundo as direções $X$ e $\theta$
$\tau_{X R} \quad$ Componente de tensão cisalhante segundo as direções $X$ e $R$
$\tau_{\theta R} \quad$ Componente de tensão cisalhante segundo as direções $\theta$ e $R$
$\tau_{\text {ini }} \quad$ Matriz de tensões iniciais 